\title{
Tendances saisonnières des alvéolites et cellulites de la face en France : données du moteur de recherche Google Trends@
}

\author{
M. Hadj SaÏd (Marseille), F. Campana (Marseille), U. Ordioni (Marseille), R. Lan \\ (Marseille), C.M. Chossegros (Marseille)
}

Introduction : Certaines pathologies présentent des tendances saisonnières dans leur incidence et leur prévalence. Ces tendances s'expriment par l'intérêt affiché dans le nombre de recherches sur Internet pour ces pathologies selon les mois de l'année. Matériel et Méthodes : Les données des requêtes de Google Trends@ ont été extraites de décembre 2007 à décembre 2017. Celles-ci ont été traitées et calculées par volume trimestriel de recherche correspondant à chacune des quatre saisons sur cette période de 10 ans. Les données ont été isolées pour la région géographique spécifique correspondant à la France pour les termes de recherche suivants : « alvéolite », « cellulite de la face », « alvéolite dentaire », « cellulite dentaire » et " abcès dentaire ». Le même procédé a été réalisé pour les régions géographiques correspondant aux USA et à l'Australie avec les termes de recherche " alveolitis », " facial cellulitis », " dental alveolitis », " dental cellulitis », « dental abcess ». Les effets saisonniers ont été calculés à l'aide de modèles de régression. Résultats : En France, le volume de recherche est le plus élevé en hiver. Les patients manifestent le plus faible intérêt de recherche pour les cellulites et abcès dentaires pendant l'été $(p<0,05)$. Les variations saisonnières sont comparables aux États-Unis (hémisphère nord) alors qu'en Australie (hémisphère sud), elles présentent une tendance inverse. Les tendances de recherche pour l'alvéolite sont stables durant l'année $(\mathrm{p}<0,05)$. Conclusion : Au regard des données des requêtes de Google Trends, les cellulites de la face semblent plus susceptibles de se produire pendant les mois d'hiver, contrairement aux alvéolites qui n'ont pas de tendance saisonnière. 